\title{
Superdense State of Metals Compressed by Shock Waves
}

\author{
R.F. Trunin \\ RFNC-VNIIEF, Sarov. Russia
}

\begin{abstract}
The paper presents explosive devices designs for generation of strong shock waves. Record values of specific internal energy of shock-compressed iron are obtained with use of them.
\end{abstract}

[compression, shock waves, energy, pressures, iron]

\section{Introduction}

The main limitation at the operation of static devices for study of substance compression is the strength of matrix material, in which the tested sample is located. Several (1-3) hundreds of GPa is the capabilities limit of static devices at these measurements.

Dynamic method is free from limits, connected with the strength of used constructions. That is why there are no limits for it at the researches progress to the area of as high as wished pressures. It is connected with wonderful property of this method, in which the kinematics parameters of shock wave - the speed of wave front propagation $D$ and speed of compressed substance motion behind the wave front $U$, play the role of piezometer. Thermodynamic values, such as pressure $P$. density $\rho$ (or specific volume $V=1 / \rho$ ) and the energy of shock compression - internal substance energy $E$ are expressed through $D$ and $U$ from the laws of conservation of pulse $P-P_{0}=D \cup N_{0}$, mass $V=V_{0}(D$ $U) / D$ and energy $E-E_{0}=0.5 P\left(V_{0}-V\right)$.

Half of this work, done by shock wave on the substance mass unit, transfers to the kinetic energy, and the other half is spent for the increasing of specific internal energy on the value $E_{1}-E_{0}=0.5 P_{1}\left(V_{0}-V_{1}\right)$. Kinematics values $D$ and $U$ are the experimental values, which are defined at the shock-wave experiments. That's why it is much more convenient to use these parameters for estimation of energetic states in compressed substances. It is clear from the mentioned above laws of conservation of energy, that kinetic energy. which is equal on its value to the internal energy, is expressed through the mass speed $U$ in the from: $E=0.5 U^{2}$. We will use this proportion for our estimation.

\section{Internal Energy of Metal Shock Compression}

\subsection{Measurements on Laboratory Devices}

There are a lot of laboratory shock waves generators, which create pressures from fractions to several hundreds of GPa at the investigated samples. Typical samples of used generators are shown on the Fig. 1-3. The scheme of so-called "flat" measurement unit is shown on the first one. After the detonation wave propagation, the explosion products, braking with the help of metal shield, which protects the investigated samples, create stationary shock wave in them. Varying the charge weight, its composition and shield material, is possible to obtain different states of shock compression at the investigated samples. For iron, for example, maximum parameters are: $P=40 \mathrm{GPa}, E=0.5$ $\mathrm{kJ} / \mathrm{g}$.

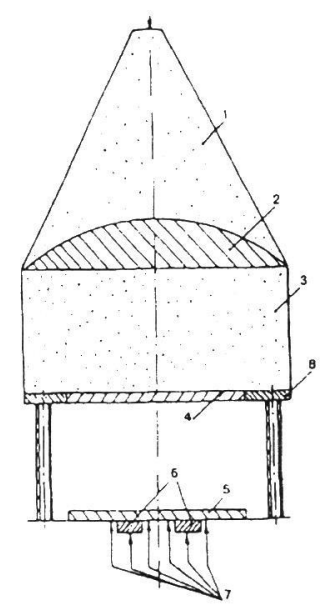

Fig. 1 The device scheme with acceleration of aluminium striker. 1 - Lens charge of explosive, 2 - Flatwave lens, 3 - Main charge, 4 -Striker, 5 - Shield from standard material. 6 - Investigated sample 7 Electrocontact probe, 8 - Support ring (Fe) 


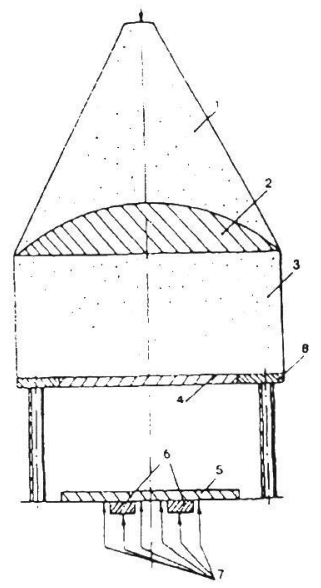

Fig. 2 The device with acceleration of steel striker. 1-7 - The same. as Fig. 1, 8 - $1 \mathrm{~mm}$ thickness Plexiglas's gasket.

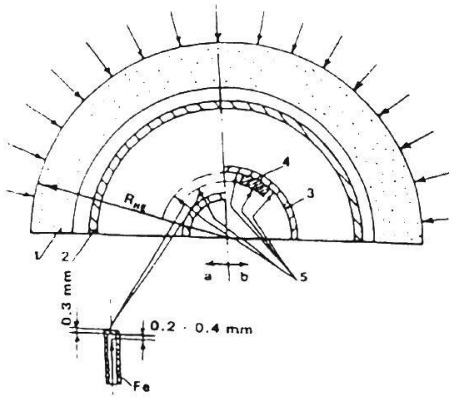

Fig. 3 The device with over compressed detonation wave. 1 - Explosive charge, 2 - Shell-striker (Fe), 3 Shield (Fe), 4 - Samples, 5 - Electrocontact probe

Fig 3 shows the scheme of shock wave generator, based on the acceleration up to different speed (maximum values, approximately, are $6.0-6.5 \mathrm{~km} / \mathrm{s}$ ) of thin metal (steel and aluminium) shock-plates. The shock of such plates on the samples creates pressures $P=200 \mathrm{GPa}$ (maximum parameters) and $E=4 \mathrm{~kJ} / \mathrm{g}$ in them

System. showed on the Fig.3, has record parameters. In this case thin iron shell is accelerated by the explosion products of convergent detonation wave. The speed of this shell is constantly growing as the shell "converges" to the center. Change of its motion speed versus the radius is shown in Fig. 4.

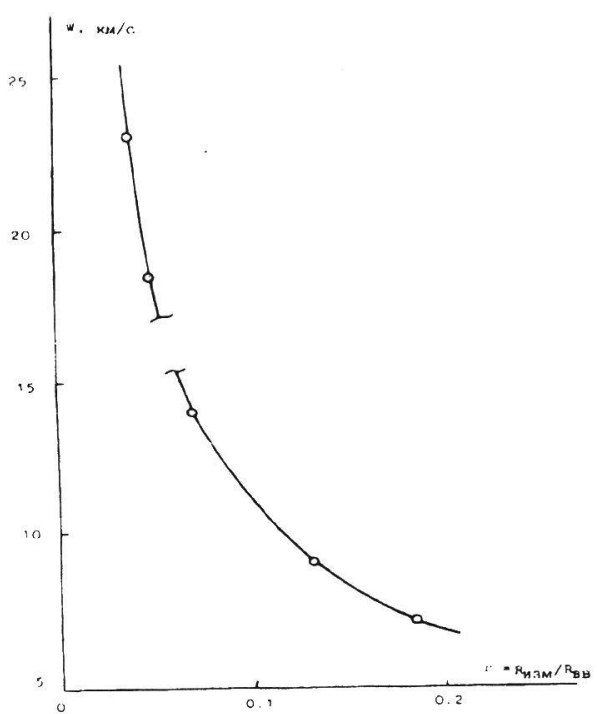

Fig. 4. Speed of projectiles in the spherical systems

On the deep radius of movement (close to the system center) the shell speed reaches $23 \mathrm{~km} / \mathrm{s}$ in direct measurements (mass speed in the samples is equal to the half of this value) and intemal energy in iron samples is $66 \mathrm{~kJ} / \mathrm{g}$, Record energy value in these systems were obtained with aluminium: $E=113 \mathrm{~kJ} / \mathrm{g}$.

Is it possible to increase the shock waves intensity at the investigated samples? Actually, small enlargement of shock waves parameters could be obtained. For this it is necessary to use more powerful explosion compositions (reserves are limited here, because powerful enough explosion substance is used in the generator, shown on the Fig.3), to use the greater weights (generator dimensions), to make measurements on the deeper radiuses and so on. Estimations show, that these measures will increase the parameters of sphere device approximately on $20 \%$, which is not equal to the expenses, which will be necessary to conduct to work out modern technology of its creation. That's why it is possible to consider the existing hemi-sphere measurement device (Fig.3) as optimum one

\section{Measurement at the Underground Nuclear Explosions 3.1. Iron Shock Compression Energy}

Significantly greater power parameters were fixed at the underground nuclear explosions, which, alas, fell into oblivion. The experimental setup on the measurement of iron compression is shown on the Fig. 5. 


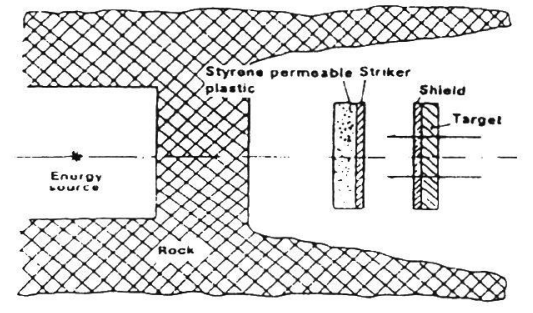

Fig. 5 Measurement scheme of kinematic parameters definition $(D, U)$ of iron at underground explosions.

The experimental device represents the system of plate acceleration by "explosion products" of cellular styrofoam (polystyrene foam) (initial density 0.03 $\mathrm{g} / \mathrm{cm}^{3}$ ). Which plays the role of softening gasket, up to the speed $36-61 \mathrm{~km} / \mathrm{s}$. The strike of the plate, flying with such speed, to the target, creates the pressure of shock compression 4.1-10.5 TPa at the investigating samples. Correspondingly, the energy of shock compression at the last case $E=470 \mathrm{~kJ} / \mathrm{g}$. Experimental connection of internal energy in iron depending on the shock waves pressure is shown on the Fig.6. It represents a broken line. consisted of two line segments of different inclination with the intersection point at $P=3.0 \mathrm{TPa}$. Maximum energy value is $470 \mathrm{~kJ} / \mathrm{g}$, that, approximately, 110 times bigger, that the explosion energy of 1 gramm of TNT

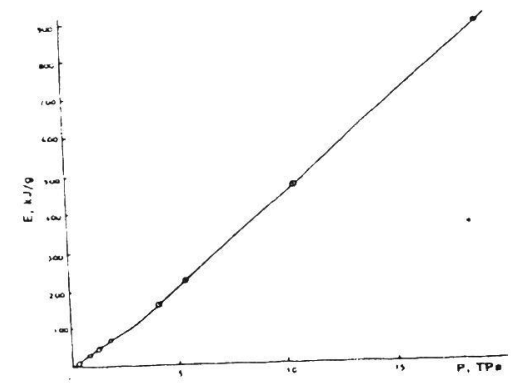

Fig. 6 The dependence of shock compression energy from pressure for iron. Absolute measurements (o laboratory and firing point measurements) - comparable measurements $(P=20 \mathrm{TPa})$.
These data, which are absolute in methodical sense, are best-possible according to the recorded parameters, including the values of internal energy. The specific energy is about $1 \mathrm{MJ} / \mathrm{g}$ in the area of reliable interpolation. which can be taken equal to the absolute methods of measurement. Concerning comparative definitions (compressibility, relative to standard, substance, adiabatic curve of which is know), in [1], where the giant speeds of shock wares, reaching $D_{\mathrm{Fe}}=90$ $\mathrm{km} / \mathrm{s}\left(\cup_{\mathrm{Fe}_{\mathrm{e}}}=70 \mathrm{~km} / \mathrm{s}\right)$ are registered, $E=2.5 \mathrm{MJ} / \mathrm{g}$ correspondingly. This is the record energy value, defined in the conditions of underground explosions.

Considered examples show, that high-power states, with energy density, comparable with loading devices based, for example on the systems with magnetic cumulation, or laser effect on the substance realize at the effect of powerfull shock waves on the samples.

\section{Reference}

[1] Avrorin E.N., Vodolaga B.K. Voloshin N.P. Kovalenko G.V. Kuropatenko VF, Simonenko V.A and Chernovolyouk B.T. Experimenta Investigations of Shell Effects on Shock Adiabats of Condensed Substances, Journal of Experimental and Theoretical Physics(ZETPh), Vol.93, No.8, 1987. P.613-622, russian

[Received 13 April 1998] 